\title{
Estado nutricional e capacidade funcional na úlcera por pressão em pacientes hospitalizados
}

\author{
Nutritional status and functional capacity \\ predispose hospitalized patients \\ to pressure ulcers
}

\author{
Francine PERRONE ${ }^{1}$ \\ Adriene Alexandra PAIVA ${ }^{1}$ \\ Letícia Martins Ignácio de SOUZA' \\ Cássia da Silva FARIA ${ }^{1}$ \\ Márcia Carolina de Siqueira PAESE ${ }^{1}$ \\ José Eduardo de AGUILAR-NASCIMENTO' \\ Diana Borges DOCK-NASCIMENTO'
}

\section{R E S U M O}

\section{Objetivo}

Correlacionar a incidência de úlcera por pressão com o estado nutricional e a capacidade funcional de pacientes internados.

\section{Métodos}

Estudo de corte transversal realizado em dois hospitais, totalizando 130 pacientes (idade mediana $=52(14-85)$ anos, 77 (59,2\%) homens e 53 (40,8\%) mulheres), sendo 72 (55,4\%) internados para tratamento clínico, 40 $(30,8 \%)$ para tratamento cirúrgico e $18(13,8 \%)$ em terapia intensiva. Os pacientes foram avaliados pela avaliação subjetiva global e classificados, de acordo com sua capacidade funcional, em acamados e não acamados. Registrou-se a presença e número de úlceras, e sua gravidade. A classificação das úlceras foi estabelecida como grave, para lesões de $3^{\circ}$ e $4^{\circ}$ graus, e leve, para os graus 1 e 2 .

\section{Resultados}

A incidência de úlcera por pressão na população estudada foi de $19,2 \%(n=25)$. Não houve associação significativa com o sexo, a idade e tipo de tratamento do paciente. Os pacientes acamados apresentaram 7,5 vezes mais chance de apresentar úlceras $(19 / 50 ; 38,0 \%)$ do que os que deambulavam (6/80; 7,5\%; OR=7,5;

${ }^{1}$ Universidade Federal de Mato Grosso, Hospital Universitário Júlio Müller, Equipe Multidisciplinar de Terapia Nutricional. R. Luís Philippe Pereira Leite, s/n., Alvorada, 78048-902, Cuiabá, MT, Brasil. Correspondência para/Correspondence to: D.B. DOCK-NASCIMENTO. E-mail: <dianadock@hotmail.com>. 
432 | F. PERRONE et al.

IC95\%: 2,7-20,7; $p<0,001)$. A incidência de úlcera nos pacientes gravemente desnutridos $(20 / 49 ; 40,8 \%)$ foi 10 vezes maior que nos pacientes considerados não gravemente desnutridos (5/81; 6,1\%; OR=10,4 IC95\%: 3,6-30,5; $p<0,0001)$ Pela análise multivariada, tanto a capacidade funcional (acamado, OR=9,2; IC95\%: $2,8-30,1 ; p<0,001)$ quanto o estado nutricional (desnutrido grave, OR=3,8; IC95\%: 1,0-13,9; $p=0,04$ ) associaram-se com a úlcera por pressão.

\section{Conclusão}

A incidência de úlcera por pressão está diretamente correlacionada com a desnutrição e com a restrição ao leito dos pacientes internados.

Termos de indexação: Capacidade residual funcional. Desnutrição. Pacientes hospitalizados. Úlcera por pressão.

\section{A B S T R A C T}

\section{Objective}

This study investigated if pressure ulcer correlated with the nutritional status and functional capacity of hospitalized patients.

\section{Methods}

This cross-sectional study included 130 patients of two hospitals, 77 (59.2\%) men and 53 (40.8\%) women. The median age of the sample was 52 (14-85) years. Seventy-two (55.4\%) patients were hospitalized for clinical treatment, 40 (30.8\%) for surgical treatment and 18 (13.8\%) for intensive care. Nutritional status was determined by subjective global assessment. The patients were then classified according to their functional capacity as bedridden or not. The number and severity of pressure ulcers was recorded. Grades 3 and 4 pressure ulcer were considered severe and grades 1 and 2 were considered mild.

\section{Results}

The incidence of pressure ulcers in the studied population was $19.2 \%(n=25)$. Pressure ulcer were not associated with gender, age and type of treatment. Bedridden patients were 7.5 times more likely to have pressure ulcer (19/50; 38.0\%) than those who could walk (6/80; 7.5\%; OR=7.5; C195\%: 2.7-20.7; $p<0.001)$. The incidence of pressure ulcers in severely malnourished patients (20/49; 40.8\%) was 10 times greater than that of better nourished patients (5/81; 6.1\%; OR=10.4 C195\%: 3.6-30.5; $p<0.0001)$. According to multivariate analysis, both functional capacity (bedridden, $O R=9.2 ; C 195 \%: 2.8-30.1 ; p<0.001$ ) and nutritional status (severe malnutrition, $O R=3.8$; C195\%: 1.0-13.9; $p=0.04$ ) are associated with pressure ulcer.

\section{Conclusion}

Pressure ulcers correlate directly with malnutrition and bedridden status in hospitalized patients.

Indexing terms: Functional residual capacity. Malnutrition. Hospitalizad patients. Pressure ulcer.

\section{N T R O D U ÇÃ O}

As Úlceras por Pressão (UP) são definidas como "área de lesão localizada da pele e dos tecidos subjacentes, causadas por pressão, tensão tangencial, fricção e/ou uma combinação destes fatores" e estão associadas com maior risco de morbidade e mortalidade ${ }^{1}$. A prevalência de úlcera por pressão varia de 3-14\% em pacientes hospitalizados de todas as idades e de $20-41 \%$ em casas de repouso e hospitais para idosos 2,3 . No Brasil, há poucos trabalhos publicados sobre o tema. Rogenski \& Santos ${ }^{4}$ relatam 38,0\% de incidência em pacientes internados em hospital universitário, com idade variando entre 22 e 95 anos. Souza \& Santos $^{5}$ encontraram 38,9\% de incidência em pacientes idosos institucionalizados.

Fatores de risco relacionados com o aparecimento de úlcera por pressão incluem a idade, imobilidade, incontinência urinária ou fecal que leva à umidade local, atrito ou força de fricção, comorbidades relacionadas com gasto metabólico elevado, doenças crônicas e deterioração do estado nutricional ${ }^{6,7}$. Dentre tais fatores, o estado nutricional e a capacidade funcional merecem destaque entre as principais causas. 
Pacientes desnutridos têm alterações metabólicas que desfavorecem a cicatrização e fragilizam os tecidos ${ }^{8}$. No contexto nacional e mundial, a incidência de desnutrição é alta; no Brasil, estima-se que $48,1 \%$ de pacientes internados estejam desnutridos ${ }^{9}$. Outro ponto relevante é que a idade, como único fator, já coloca o paciente em risco nutricional. Isso foi confirmado no estudo de Félix \& Souza ${ }^{10}$, que observaram um risco nutricional em $83,8 \%$ dos idosos quando considerado pelo menos um dos índices antropométricos abaixo da normalidade, e 75,7\% de risco quando a miniavaliação nutricional foi empregada. Rauen et al. ${ }^{11}$ também encontraram uma alta prevalência $(45,5 \%)$ de idosos com baixo peso, em instituição geriátrica, utilizando o Índice de Massa Corporal ${ }^{12}$.

A condição funcional do paciente acamado também é importante fator de risco. A imobilidade do paciente no leito ocasiona uma força de pressão que resulta em isquemia tecidual, dificultando a liberação de oxigênio e levando ao acúmulo de metabólitos e edema. Todos esses fatores, em conjunto, podem levar ao aparecimento da úlcera por pressão ${ }^{13}$.

Entretanto, segundo a American Society for Parenteral and Enteral Nutrition (ASPEN), apesar de haver uma relação forte entre desnutrição e úlceras por pressão, ainda há carência de estudos mostrando uma conclusiva relação causal ${ }^{14}$. Nesse contexto, novos trabalhos de incidência e de correlação para UP são necessários, principalmente no Brasil. Assim, realizou-se o presente estudo com o objetivo de estudar a associação da presença de UP com o estado nutricional e a capacidade funcional em pacientes internados.

\section{MÉ T O D O S}

Estudo de corte transversal realizado no mês de dezembro de 2008 nas enfermarias e Unidades de Terapia Intensiva (UTI) de dois hospitais públicos de alta complexidade, em Cuiabá (MT). Foram estudados 130 pacientes (idade mediana $=52$ (14-85) anos, 77 (59,2\%) homens e $53(40,8 \%)$ mulheres), internados para tratamento clínico $(n=72 ; 55,4 \%)$, tratamento cirúrgico
( $n=40 ; 30,8 \%)$ ou cuidados críticos em UTI ( $n=18$; $13,8 \%)$.

Todos os pacientes foram avaliados pela Avaliação Subjetiva Global (ASG) segundo Detsky et al. ${ }^{15}$ por nutricionistas da Equipe Multidisciplinar de Terapia Nutricional (EMTN) previamente treinadas. Para fins de análise estatística, os pacientes foram posteriormente divididos em desnutridos graves (ASG-C) e não desnutridos graves (ASG-A e ASG-B).

Os pacientes foram classificados de acordo com sua capacidade funcional em acamados e não acamados.

Registrou-se a presença ou não de UP, assim como sua quantidade e gravidade. A gravidade da UP foi estabelecida de acordo com a escala elaborada pela European Pressure Ulcer Advisory Panel (EPUAP) 1 (Quadro1). Úlceras graves foram rotuladas como lesões de $3^{\circ}$ e $4^{\circ}$ graus, e leves, classificadas como lesões em graus 1 e 2 .

Utilizou-se o teste do qui-quadrado ou o de Fisher para comparar proporções de pacientes com e sem UP. O teste $t$ de Student foi utilizado para comparar variáveis contínuas entre os grupos. Utilizou-se a análise de regressão logística univariada e multivariada para determinar a força de associação entre o estado nutricional e a capacidade funcional com a presença de UP. Estabeleceu-se em 5\% $(p<0,05)$ o nível de significância estatística. Os dados foram avaliados estatisticamente através do programa SPSS 11.0.

O estudo foi aprovado pelo Comitê de Ética em Pesquisa do Hospital Universitário Júlio Muller, com número de processo n 596/CEP-HU JM/08, e seguiu os princípios éticos contidos na Declaração de Helsinki (2000), além do atendimento a legislações específicas do País. Todos os participantes assinaram um Termo de Consentimento livre e esclarecido antes de sua inclusão na amostra.

\section{RES U LT A D O S}

A incidência de UP na população estudada foi de 19,2\% ( $n=25)$. No Quadro 1 encontram-se 
a classificação e a distribuição das UP de acordo com a gravidade. A maioria dos 25 pacientes apresentava apenas uma UP $(n=17 ; 68 \%)$. Seis $(24,0 \%)$ pacientes apresentavam duas lesões, um apresentava três (4,0\%) e outro, quatro $(4,0 \%)$ lesões. Noventa e três $(71,5 \%)$ dos pacientes receberam terapia nutricional por via oral e $37(28,5 \%)$ receberam terapia enteral. Dos pacientes com diagnóstico de UP $(n=25), 17(68,0 \%)$ estavam recebendo terapia enteral e $8(32,0 \%)$, dieta via oral.

Na Tabela 1 pode ser vista a análise univariada de diversas variáveis clínicas com a ocorrência de UP. Observa-se que não houve associação significativa com o sexo, idade e local de internação do paciente. Embora sem diferença significativa, pacientes internados em UTI apresentaram uma chance de ter UP 2,4 vezes maior que os internados em enfermarias (OR: 2,4, IC $95 \%$ : 0,8-7,3; $p=0,11)$.

\section{Capacidade funcional}

Os pacientes acamados perfizeram 38,5\% $(n=50)$ da casuística. Na população com ulcera por pressão $76,0 \%$ eram acamados e $24 \%$ deam-

Quadro 1. Classificação utilizada e números de casos de úlceras por pressão na população estudada. Cuiabá (MT), 2008.

\begin{tabular}{|c|c|c|c|}
\hline \multirow{2}{*}{ Estágio } & \multicolumn{2}{|c|}{ Casos } & \multirow{2}{*}{ Característica } \\
\hline & $\mathrm{n}$ & $\%$ & \\
\hline Grau 1 & 2 & 8 & $\begin{array}{l}\text { Eritema não reversível da pele intacta. Descoloração da pele, calor, edema e endurecimento podem tam- } \\
\text { bém ser usados como indicadores, particularmente em indivíduos com pele mais escura. }\end{array}$ \\
\hline Grau 2 & 6 & 24 & $\begin{array}{l}\text { Perda parcial da pele, envolvendo epiderme, derme ou ambas. A úlcera é superficial e apresenta-se clini- } \\
\text { camente como um abrasão ou bolha. }\end{array}$ \\
\hline Grau 3 & 12 & 48 & $\begin{array}{l}\text { Perda total da espessura da pele, envolvendo necrose do tecido subcutâneo, que pode estender-se até a } \\
\text { fáscia muscular subjacente, porém sem atingi-la totalmente. }\end{array}$ \\
\hline Grau 4 & 5 & 20 & $\begin{array}{l}\text { Destruição extensa, necrose dos tecidos, lesão muscular, óssea ou das estruturas de suporte, com ou sem } \\
\text { perda da estrutura total da pele. }\end{array}$ \\
\hline
\end{tabular}

Fonte: European Pressure Ulcer Advisory Panel1.

Tabela 1. Análise univariada da ocorrência de úlcera por pressão segundo diversas variáveis clínicas. Cuiabá (MT), 2008.

\begin{tabular}{|c|c|c|c|c|c|}
\hline \multirow{3}{*}{ Variável clínica } & \multicolumn{4}{|c|}{ Úlcera de pressão } & \multirow{3}{*}{$p$} \\
\hline & \multicolumn{2}{|c|}{ Sim } & \multicolumn{2}{|c|}{ Não } & \\
\hline & $n$ & $\%$ & $\mathrm{n}$ & $\%$ & \\
\hline \multicolumn{6}{|l|}{ Capacidade funcional } \\
\hline Acamado & 19 & 38,0 & 31 & 62,0 & \multirow{2}{*}{$<0,001$} \\
\hline Não acamado & 6 & 7,5 & 74 & 92,5 & \\
\hline \multicolumn{6}{|l|}{ Desnutrição grave } \\
\hline $\operatorname{sim}$ & 20 & 40,8 & 29 & 59,2 & \multirow[t]{2}{*}{$<0,001$} \\
\hline Não & 5 & 6,2 & 76 & 93,8 & \\
\hline \multicolumn{6}{|l|}{ Sexo } \\
\hline Masculino & 13 & 16,9 & 64 & 83,1 & \\
\hline Feminino & 12 & 22,6 & 41 & 77,4 & 0,49 \\
\hline Idade (mediana e variação) & 46 & $17-85$ & 53 & $14-83$ & 0,34 \\
\hline \multicolumn{6}{|l|}{ Diagnóstico } \\
\hline Clínico & 13 & 18,1 & 59 & 81,9 & \\
\hline Cirúrgico & 6 & 15,0 & 34 & 85,0 & \multirow[b]{2}{*}{0,24} \\
\hline Crítico & 6 & 33,3 & 12 & 66,7 & \\
\hline Total & 25 & 19,2 & 105 & 80,8 & \\
\hline
\end{tabular}


bulavam, como pode ser visto na Figura 1. Os pacientes acamados apresentaram 7,5 vezes mais chance de ter UP $(19 / 50 ; 38,0 \%)$ do que os que deambulavam (6/80; 7,5\%; OR=7,5 IC95\% 2,7$20,7 ; p<0,001)$. Pacientes acamados apresentaram significativamente $(p<0,001)$ mais lesões classificadas como graves, quando comparados com os não acamados (Tabela 2).

\section{Estado nutricional}

Pela ASG, 49 (37,7\%) pacientes foram considerados como gravemente desnutridos (ASG-C), 50 (38,5\%) como desnutridos moderados (ASG-B) e $31(23,8 \%)$ como eutróficos (ASG-A). A chance de ocorrer UP nos pacientes gravemente desnutridos (20/49; 40,8\%) foi aproximadamente 10 vezes maior que nos pacientes considerados como não gravemente desnutri$\operatorname{dos}(5 / 81 ; 6,1 \%$; OR=10,4 IC95\%: 3,6-30,5; $p<0,0001)$ como pode ser visto na Figura 2. Pacientes com desnutrição grave apresentaram significativamente $(p<0,001)$ mais lesões classificadas como graves (Tabela 3). Cerca de 88,0\% dos casos avançados de UP foram vistos em pacientes com desnutrição grave

Pela análise multivariada, tanto a capacidade funcional (acamado, OR=9,2; IC95\%: 2,8-30,1; $p<0,001)$ quanto o estado nutricional (desnutrido grave, $O R=3,8$; IC95\%: 1,0-13,9; $p=0,04)$ associaram-se significantemente com a UP.

Tabela 2. Estadiamento da úlcera por pressão e associação com capacidade funcional e desnutrição grave. Cuiabá (MT), 2008.

\begin{tabular}{|c|c|c|c|c|c|}
\hline \multirow{3}{*}{ Variável } & \multicolumn{4}{|c|}{ Gravidade de úlcera por pressão } & \multirow{3}{*}{$p$} \\
\hline & \multicolumn{2}{|c|}{ Leve } & \multicolumn{2}{|c|}{ Grave } & \\
\hline & $\mathrm{n}$ & $\%$ & $\mathrm{n}$ & $\%$ & \\
\hline \multicolumn{6}{|c|}{ Capacidade funcional } \\
\hline Acamado & 36 & 72,0 & 14 & 28,0 & \\
\hline Não acamado & 77 & 96,0 & 3 & 4,0 & $<0,001$ \\
\hline \multicolumn{6}{|c|}{ Desnutrição grave } \\
\hline Sim & 34 & 69,4 & 15 & 30,6 & \\
\hline Não & 79 & 97,5 & 2 & 2,5 & $<0,001$ \\
\hline
\end{tabular}

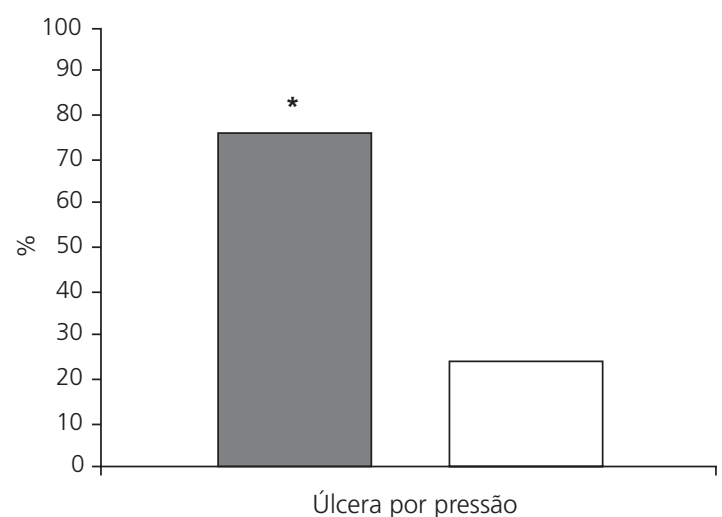

$\square$ Acamados $\square$ Não acamados

Figura 1. Incidência de úlcera por pressão de acordo com a capacidade funcional. Cuiabá (MT), 2008

Nota: * $p<0,001$ versus não acamados.

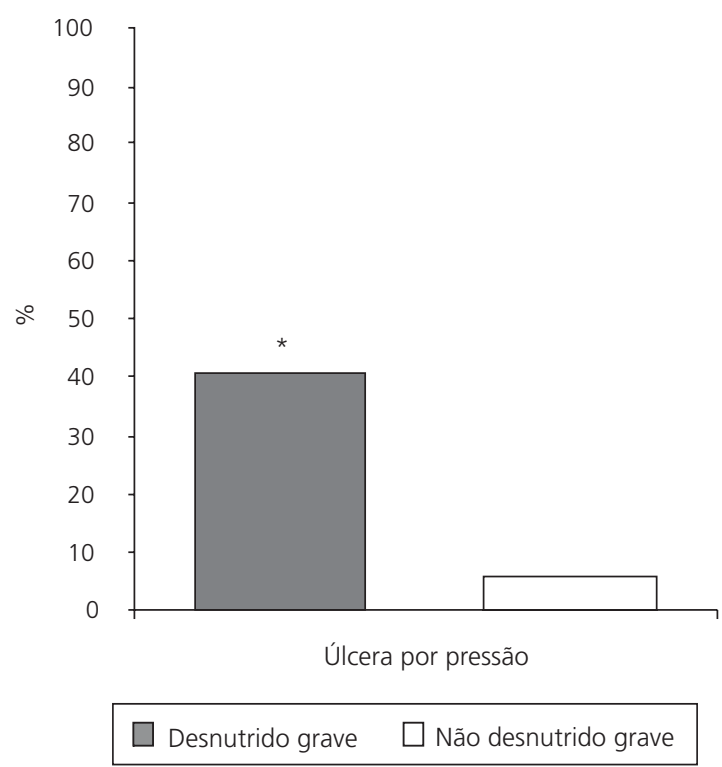

Figura 2. Frequência de úlcera por pressão em pacientes considerados desnutridos graves (ASG-C) ou não gravemente desnutridos (ASG-A e ASG-B). Cuiabá (MT), 2008.

Nota: * $p<0,0001$ versus não gravemente desnutridos.

\section{DISCUSS ÃO}

Os resultados mostraram uma alta correlação da presença de UP na população desnutrida e com capacidade funcional reduzida. Foi inte- 
ressante perceber que a relação com o estado nutricional só existiu em pacientes considerados gravemente desnutridos. Nessa população também ocorreram os casos mais avançados de úlcera de pressão. Isso sugere que o estado nutricional e a capacidade funcional têm um papel importante na gênese das UP e uma nítida relação com a sua gravidade.

Há forte evidencia na literatura demonstrando que a prevalência no desenvolvimento da UP em pacientes hospitalizados e idosos é alta. Estudos envolvendo grandes casuísticas com número superior a 10 mil casos mostram uma incidência de $28-41 \%$ de UP em idosos em hospitais europeus ${ }^{16}$. No presente trabalho, não se encontrou uma relação estatística com a idade. Provavelmente isso pode ter ocorrido devido ao número de casos estudados ter sido pequeno em relação a outros trabalhos com maior casuística, e também, pelo fato de a idade média dos pacientes avaliados ser de 50 anos. Além disso, o estudo foi realizado em hospital de alta complexidade (hospital universitário), acarretando um possível viés de confundimento. Entretanto, os resultados estão em concordância com Souza \& Santos $^{5}$ e Chacon et al. ${ }^{17}$ e, que também não encontraram correlação entre idade e prevalência de UP.

A UTI, pelo grau reduzido de capacidade funcional dos pacientes internados, é uma unidade de internação que apresenta alta prevalência de UP1,3,13,18. No Brasil, Louro et al. ${ }^{18}$ estudando 155 pacientes internados em UTI, encontraram 18 pacientes que já apresentavam UP no momento da admissão, e outros 40 pacientes que vieram a desenvolver a UP durante a internação, perfazendo uma incidência de $25,8 \%{ }^{18}$. Também em estudo nacional, Blanes et al. ${ }^{19}$ encontraram uma prevalência de $19,2 \%$ de pacientes com UP em unidade de terapia intensiva. Neste estudo, ocorreu o dobro de UP entre pacientes críticos, quando comparados a pacientes em enfermarias. Entretanto, a análise estatística mostrou que essa diferença não foi significativa, provavelmente também pelo número de casos estudados.

A alta incidência de desenvolvimento de UP no período intra-hospitalar normalmente é justificada pela condição comprometida da capacidade funcional, que determinou o estado acamado dos pacientes ${ }^{13}$. Louro et al. ${ }^{18}$ mostraram que, nos casos novos, o aparecimento da UP deu-se por volta do $7^{\circ}$ dia de internação na UTI. Consistentemente com a literatura, os achados deste estudo mostraram que $38 \%$ dos pacientes que não possuíam autonomia para deambular apresentaram UP durante o período de internação. Nessa linha de ideias, Coelho \& Silva ${ }^{20}$ relataram que a mudança de decúbito, entre outros fatores, está diretamente relacionada com a presença de um acompanhante, sendo que, quando este se fez presente, $100 \%$ dos pacientes apresentaram melhora do estágio de úlcera. Outro ponto importante a ser sinalizado é que a presença de UP por si só já influencia negativamente na qualidade de vida de pacientes internados por longo tempo, em casas de repousos ${ }^{21}$.

Além da imobilidade, o estado nutricional prévio, a atenção nutricional durante a hospitalização e o atendimento dispensado pela enfermagem são também fatores predisponentes para UP22. De fato, alguns estudos têm demonstrado uma relação causal direta entre a nutrição e o desenvolvimento desse tipo de lesão $0^{2,3,5,6,8,8,18,22}$. Dentre os nutrientes que mais influenciam na prevenção e tratamento das UP, merecem destaque a proteína, o aminoácido arginina, o zinco e a vitamina $\mathrm{C}^{23}$. Sabidamente, o estado nutricional interfere na qualidade da cicatrização e influencia diretamente a relação anabolismo e catabolismo ${ }^{14}$. Além disso, a desnutrição pode influenciar na vulnerabilidade dos tecidos aos fatores extrínsecos relacionados com a gênese da UP, como por exemplo, a pressão' ${ }^{1}$ Confirmando essa tese, Fife et al. ${ }^{24}$ demonstraram que a UP pode ocorrer já na primeira semana após a internação em UTI quando os pacientes apresentam baixo peso e baixo índice de massa corporal na admissão. Allman et al. ${ }^{25}$ também encontraram associação entre o baixo peso corporal presente na admissão hospitalar e a presença de UP durante o período de hospitalização. Em concordância, os dados deste estudo mostraram que quase a metade dos pacientes 
gravemente desnutridos, com ASG-C, apresentou úlcera de decúbito. Dessa forma, Castilho \& Ca$\mid a r^{26}$, em seu trabalho de revisão, concluem que pacientes que apresentam risco para o desenvolvimento de UP podem ser identificados precocemente, através da avaliação nutricional, evitando maiores transtornos e custos hospitalares.

Vários estudos têm demonstrado que há realmente uma associação entre o desenvolvimento de UP e a imobilidade no leito ${ }^{16,20,22}$ e o estado nutricional de pacientes internados ${ }^{7,25}$. Nesse contexto, uma recente meta-análise mostrou que o suporte nutricional com dieta hiperproteica pode prevenir o aparecimento de UP em pacientes hospitalizados ${ }^{27}$. O guidelines da European Pressure Ulcer Advisory Panel and National Pressure Ulcer Advisory Panel - EPUAP e NEPUAP aconselha que pacientes em risco de desenvolver UP, mesmo recebendo dieta via oral, beneficiam-se com a prescrição de suplementos hiperproteicos ${ }^{23}$.

Os resultados permitem concluir que a incidência de úlcera de pressão está diretamente correlacionada com a desnutrição do paciente internado e sua restrição ao leito.

\section{COLABORADORES}

F. PERRONE, A.A. PAIVA, L.M.I. SOUZA, C.S. FARIA e M.C.S. PAESE participaram coleta dos dados e da elaboração do artigo. J.E.A. NASCIMENTO e D.B. DOCK-NASCIMENTO foram responsáveis pela concepção e desenho do estudo; montagem, análise de dados e elaboração e aprovação da versão final do artigo.

\section{REFERÊ N CIAS}

1. European Pressure Ulcer Advisory Panel. Pressure ulcer treatment guidelines, 2004. Available from: <http://www.epuap.org/gltreatment.html>.

2. Kerstetter JE, Holthausen BA, Fitz PA. Malnutrition in the institutionalized older adult. J Am Diet Assoc. 1992; 92(9):1109-16.

3. Allman RM, Laprade CA, Noel LB. Pressure sores among hospitalized patients. Ann Intern Med. 1986;105(3):37-42.
4. Rogenski NMB, Santos VLCG. Estudo sobre a incidência de úlceras por pressão em um hospital universitário. Rev Latino-Am Enfermagem 2005; 13(4): 474-80. doi:10.1590/S0104-11692005000400 003.

5. Souza DMST, Santos VLCG. Risk factors for pressure ulcer development in institutionalized elderly. Rev Latino-Am Enfermagem. 2007; 15(5):958-964. doi: 10.1590/S0104-11692007000500012.

6. Mathus-Vliegen EH. Old age, malnutrition, and pressure sores: an ill-fated alliance. J Gerontol. 2004; 59(4):355-60.

7. Russell L. Physiology of the skin and prevention of pressure sores. Br J Nurs. 1998; 7(18):1084-100.

8. Thomas DR. Improving outcome of pressure ulcers with nutritional interventions: a review of the evidence. Nutrition. 2001; 17(2):121-5. doi:10.10 16/S0899-9007(00)00514-1

9. Waitzberg DL, Caisffa WT, Correa MI. Hospital malnutrition: the Brazilian national survey (IBRANUTRI): a study of 4000 patients. Nutrition. 2001; 17(7-8):573-80. doi:10.1016/50899-9007 (01)00573-1.

10. Félix LN, Souza EMT. Avaliação nutricional de idosos em uma instituição por diferentes instrumentos. Rev Nutr. 2009; 22(4):571-80. doi: 10.1590/S141 5-52732009000400012.

11. Rauen MS, Moreira EAM, Calvo MCM, Lobo AS. Avaliação do estado nutricional de idosos institucionalizados. Rev Nutr. 2008; 21(3):303-10. doi: 10.15 90/S1415-52732008000300005.

12. Garcia ANM, Romani SAM, Lira PIC. Indicadores antropométricos na avaliação nutricional de idosos: um estudo comparativo. Rev Nutr. 2007; 20(4): 371-8. doi:10.1590/S1415-52732007000400004.

13. Bryant RA. An introduction to acute and chronic wound care: nursing management. J ET Nurs. 1992; 19(2):38-9.

14. American Society for Parenteral and Enteral Nutrition. Nutrition support core curriculum: a casebased approach: the adult patient. Silver Spring (MDP): ASPEN; 2007.

15. Detsky AS, McLaughlin JR, Baker JP, Johnston N, Whittaker S, Mendelson RA, et al. What is subjective global assessment of nutritional status? J Parenter Enteral Nutr. 1987; 11(1)8-13. doi: 10.117 7/014860718701100108

16. Tannen A, Dassen T, Halfens R. Differences in prevalence of pressure ulcers between the Netherlands and Germany: associations between risk, prevention and occurrence of pressure ulcers in hospitals and nursing homes. J Clin Nursing. 2008; 17(9):1237-44. doi:10.1111/j.1365-2702.20 $07.02225 x$. 
17. Chacon JM, Blanes L, Hochman B, Ferreira LM. Prevalence of pressure ulcers among the elderly living in long-stay institutions in São Paulo. Sao Paulo Med J. 2009; 127(4):211-5. doi: 10.1590/S1 516-31802009000400006.

18. Louro M, Ferreira M, Póvoa P. Avaliação de protocolo de prevenção e tratamento de úlceras de pressão. Rev Bras Ter Intensiva. 2007; 19(3):337-41. doi: 10.1590/S0103-507X2007000300012.

19. Blanes L, Duarte IS, Calil JA, Ferreira LM. Avaliação clínica e epidemiológica das úlceras por pressão em pacientes internados no Hospital São Paulo. Rev Assoc Med Bras. 2004; 50(2):182-7. doi: 10.15 90/S0104-42302004000200036.

20. Coellho SC, Silva RC. Perfil dos pacientes hospitalizados com úlcera de pressão. Rev Bras Nutr Clin. 2004; 19(2):64-9.

21. Thein HH, Gomes T, Krahn MD, Wodchis WP. Health status utilities and the impact of pressure ulcers in long-term care residents in Ontario. Qual Life Res. 2010; 19(1):81-9. doi:10.1007/s11136-009-9563-2.

22. Holm B, Mesch L, Ove H. Importance of nutrition for elderly persons with pressure ulcers or a vulnerability for pressure ulcers: a systematic literature review. Aus J Adv Nurs. 2007; 25(1):77-84.

23. European Pressure Ulcer Advisory Panel and National Pressure Ulcer Advisory Panel. Prevention and treatment of pressure ulcers: quick reference guide. Washington (DC): National Pressure Ulcer Advisory Panel; 2009.

24. Fife C, Otto G, Capsuto EG, Brandt K, Lyssy K, Murphy $\mathrm{K}$, et al. Incidence of pressure ulcers in a neurologic intensive care unit. Crit Care Med. 2001; 29(2):283-90. doi:10.1097/00003246-20010200 $0-00011$.

25. Allman RM, Goode PS, Patrick MM. Pressure ulcer risk factors among hospitalized patients with activity limitation. JAMA. 1995; 273(11):865-70. doi: 10.1001/jama.273.11.865.

26. Castilho LD, Calari MHL. Úlcera de pressão e estado nutricional: revisão da literatura. Rev Bras Enferm. 2005; 58(5):597-601. doi: 10.1590/S0034-716720 05000500018.

27. Stratton RJ, Ek AC, Engfer M, Moore Z, Rigby P, Wolfe $R$, et al. Enteral nutritional support in prevention and treatment of pressure ulcers: a systematic review and meta-analysis. Ageing Res Rev. 2005; 4(3):422-50. doi: 10.1016/j.arr.2005.0 3.005 .

Recebido em: 16/2/2009

Versão final reapresentada em:13/7/2010

Aprovado em: 21/2/2011 